
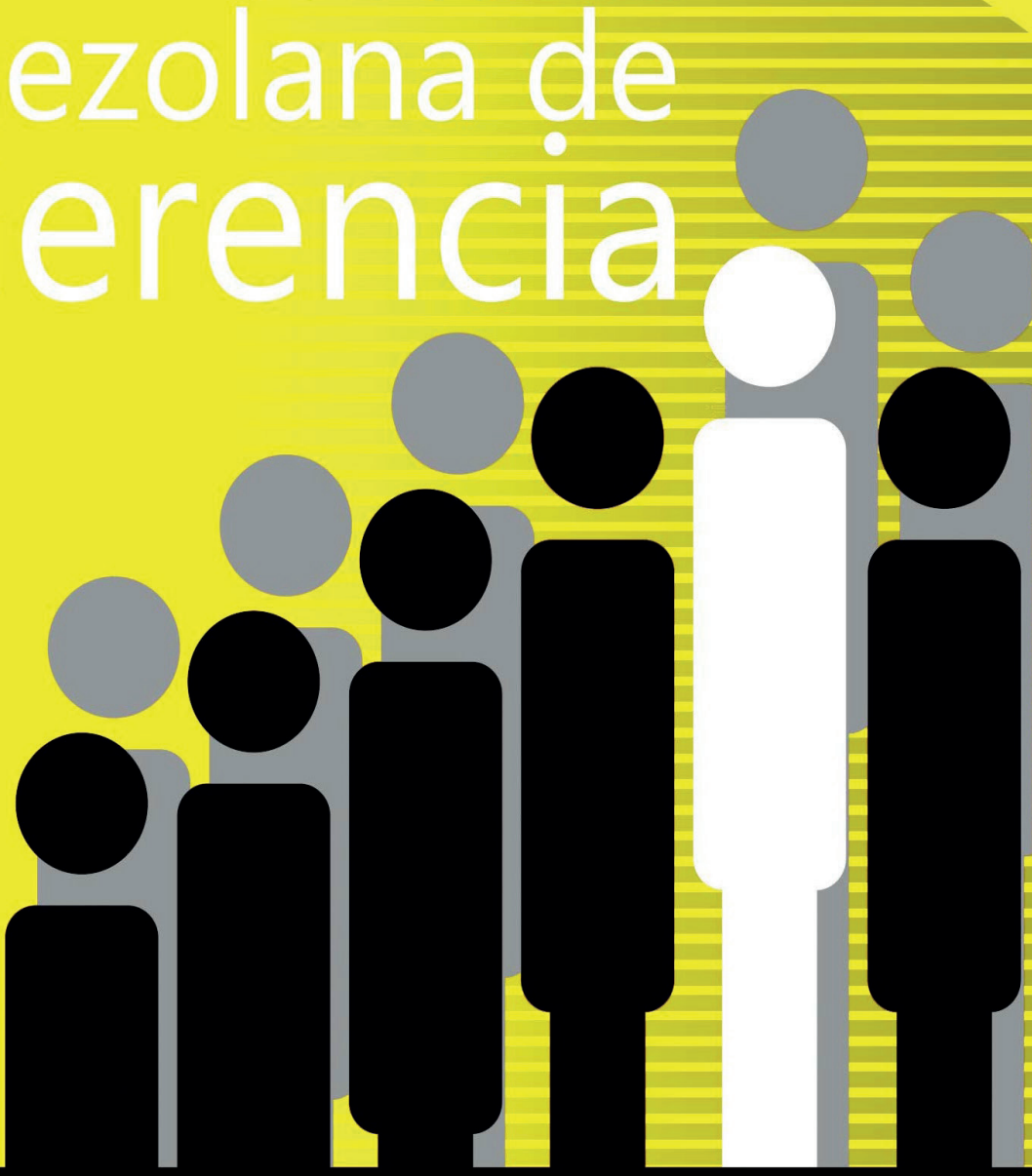


\title{
Perspectiva espacio - territorio en la gestión integral e inteligente de destinos turísticos ${ }^{*}$
}

\author{
Font Aranda, Mabel ${ }^{\star *}$
}

\begin{abstract}
Resumen
Los desplazamientos humanos han estado presentes a lo largo de la historia, y es un elemento distintivo de la actividad turística. Las personas se sienten atraídas por recursos, productos, ofertas, experiencias; naturales y culturales que existen fuera del lugar de residencia habitual. Emerge un destino turístico, donde se configuran todas las condiciones para recibir a un visitante. El objetivo es, determinar los aspectos distintivos de la gestión integral e inteligente de destinos turísticos, asumiendo como foco la perspectiva espacial-territorial. Se emplea un enfoque descriptivo y los métodos analítico-sintético, comparación, modelación esquemática a partir de la revisión bibliográfica de bases de datos internacionales. La estancia vivida en Islas Baleares, España; permite enriquecer la teoría una ejemplificación descriptiva. Se sistematizan los preceptos teórico-metodológicos, los vínculos entre las categorías referidas y los procesos que tienen lugar. Se concluye que, la perspectiva espacial (límites de nexos naturaleza sociedad) y territorial (límites de gobernanza político administrativa) junto a la calidad de las relaciones múltiples y la aplicación de los avances tecnológicos, configuran los aspectos clave de gestión integrada e inteligente que garantizan: calidad de vida a los residentes, experiencias a los turistas y sostenibilidad al destino.
\end{abstract}

Palabras clave: Gestión de destinos turísticos; destinos inteligentes; gestión integral; espacio turístico; territorio.

Recibido: 15.11 .20 aceptado: 20.02 .21

* Se agradece a la Fundación Carolina por el financiamiento de la estancia corta de investigación. A la Universidad de las Islas Baleares y a los doctores Juana María Petrus Bey y Mauricio Ruiz Pérez, quienes fueron asesores

** Posdoctorada en Gestión Integrada e Inteligente de destinos turísticos y SIG. Doctora en Ciencias Geográficas. Master en Gestión Turística. Mención Comercialización. Master en Análisis Ambiental y Ordenamiento Geoecológico. Profesora Titular Principal de la Universidad Laica Eloy Alfaro de Manabí. Ecuador. Email: mabelfont@yahoo.es ORCID: https://orcid.org/0000$\underline{0002-8207-7321}$ 


\title{
Spatial-territorial perspective in the integral and smart management of tourist destinations
}

\begin{abstract}
Human displacement has been present throughout history, and is a distinctive element of tourist activity. People are attracted to resources, products, offers, experiences; natural and cultural that exist outside the habitual place of residence. A tourist destination emerges, where all the conditions to receive a visitor are configured. The objective is; determine the distinctive aspects of the integral and intelligent management of tourist destinations, taking as a focus the spatial-territorial perspective. A descriptive approach and analytical-synthetic methods, comparison, schematic modeling is used from the bibliographic review of international databases. The stay lived in the Balearic Islands, Spain; descriptive exemplification allows enriching the theory. Theoretical-methodological precepts, the links between the referred categories and all the processes that take place are systematized. It is concluded the spatial perspective (limits of nature-society links) and territorial (limits of political-administrative governance) together with the quality of multiple relationships and the application of technological advances, configure the key aspects of integrated and smart management that guarantee: quality of life to residents, experiences to tourists and sustainability to the destination.
\end{abstract}

Key words: Management of tourist destinations; smart destinations; integral management; tourist space; territory.

\section{Introducción}

A pesar de pandemias y crisis, la actividad turística mantendrá su importancia, porque las personas requieren descansar, interactuar con familiares y amigos, estudiar, vigilar la salud o mejorarla, participar en eventos, entre otros; y varios se cumplen a través del turismo. De ahí, los desplazamientos y el desarrollo de los medios de transportación que garantizan la movilidad. A la par, comer, dormir, distraerse; son necesidades insustituibles y nicho para la proliferación de empresas afines. También, determinados recursos generan atracción y provocan movimientos hacia ellos. Esta es, la base elemental para comprender qué son los destinos turísticos y las exigencias de su gestión desde una perspectiva espacial - territorial.

El espacio manifiesta la síntesis de las relaciones naturaleza- sociedad con expresión homogénea localizable, factible de repetirse en diferentes lugares, pero con peculiaridades (Santos,1996; Mateo, 1999; Castellanos, 1994 citado por Font, 2002:21; Barrado, 2011:4; García, 2015:235). Los territorios se asumen aquí, como límites de poder político 
administrativos (Suarez et al, 2002:37; Sánchez-Crispín,

Alvarado-Sizzo, Propin-Frejomil, 2018:3; Simancas, Peñarrubia y Temes, 2018:437).

Con lo anterior se asocian los destinos turísticos, para la Organización Mundial del Turismo (OMT), es un espacio físico, con delimitación administrativa o no, donde pernocta un visitante. Agrupa productos, servicios, actividades y experiencias, en la cadena de valor del turismo, y es unidad básica de análisis del sector. Incorpora los agentes y puede extender redes hasta formar destinos mayores. Es inmaterial, con una imagen y una identidad que influye en su competitividad (OMT, 2019:15). Se requiere entonces la colaboración entre los organismos que planifican y gestionan.

En el destino, como en el espacio turístico los componentes demanda, oferta y operadores, forman un sistema de partes interconectadas (Sancho et al, 1998:47), donde se destacan las comunidades anfitrionas. La armonía entre los implicados, se materializa a través de una gestión integral; que para la OMT (2019:10) "coordina los elementos que conforman el destino". El turista percibe una experiencia global y para satisfacerlo se requiere una gestión integral (Bigné, Font y Andreu, 2000:31; Jeong \& Shin, 2020:5). La gestión inteligente, añade las tecnologías de la información y la comunicación (TIC), que acoge turistas, comunidades y gestores inteligentes.

La gestión integral e inteligente representa una nueva etapa en del "ciclo de vida de los destinos turísticos" de Butler (1980:7) en el itinerario del consumo del turista, planteado por la Secretaría de Estado de Turismo de España (SETUR, 2019:3). Para esto, los sistemas de información geográfica (SIG), integran datos necesarios para la gestión, facilita el registro, análisis, procesamiento, así como la representación gráfica ligada al espacio y territorio, dando respuesta al dónde.

España es un referente mundial de gestión integrada e inteligente. El Sistema Integral de Calidad Turística Española en Destinos (SICTED), incluye todos los servicios que se ofrecen en un destino turístico. Obtiene, mide y analiza, indicadores; para un posicionamiento competitivo presente y futuro (SETUR, 2019:3). A la par, el proyecto "Sociedad Estatal para la Gestión de la Innovación y las Tecnologías Turísticas" (SEGITTUR) se enfoca en los destinos turísticos inteligentes o smart destination. Ambos, buscan la satisfacción plena de los turistas, las comunidades y los gestores, la protección del patrimonio natural cultural y su atractividad, la sostenibilidad global (SETUR - SEGITTUR, 2018:34).

Aspectos que caracterizan a SICTED y SEGITTUR, con una configuración diferente, pueden ser aplicados a otros contextos. A tal propósito, el artículo muestra parte de la sistematización teórica, del informe de investigación "Gestión integrada e inteligente de destinos turísticos y SIG", desarrollada en la Universidad de las Islas Baleares y financiada por la Fundación Carolina. Por lo cual, el objetivo es determinar los aspectos distintivos de la gestión integral e inteligente de destinos turísticos, asumiendo como foco la perspectiva espacial-territorial.

Se emplea un enfoque descriptivo y los métodos analítico-sintético, comparación, modelación a partir de la revisión bibliográfica de bases de datos internacionales. Con el software VOSviewer se identifican los nexos conceptuales más abordados en Scopus, hasta enero de 2020. E 
Perspectiva espacio - territorio en la gestión integral e inteligente de destinos turísticos

artículo se estructura siguiendo el orden conceptual del título; además la estancia investigativa en España, permite la ejemplificación.

\section{Síntesis de la perspectiva espacio - territorio en turismo y Sistemas de Información Geográficos}

El carácter espacial y territorial del turismo se justifica porque la causa fundamental de su desarrollo son los desplazamientos que los visitantes realizan desde sus lugares de orígenes.
Existen unos atractivos turísticos, fuera del espacio habitual del visitante que incitan al disfrute y desarrollo de experiencias; para consumirlos es necesario llegar hasta ellos. El espacio geográfico, plantean Vera et al, (1997:61) es soporte, recurso y factor a la vez por su condición geográfica: magnitud espacial y atributos de valor intrínseco (natural o cultural). La valoración histórica conduce a la "adecuación" de recursos en atractivos, en producto y oferta; objeto de venta y consumo turístico (diagrama 1).

\section{Diagrama 1 \\ Espacialidad del turismo}

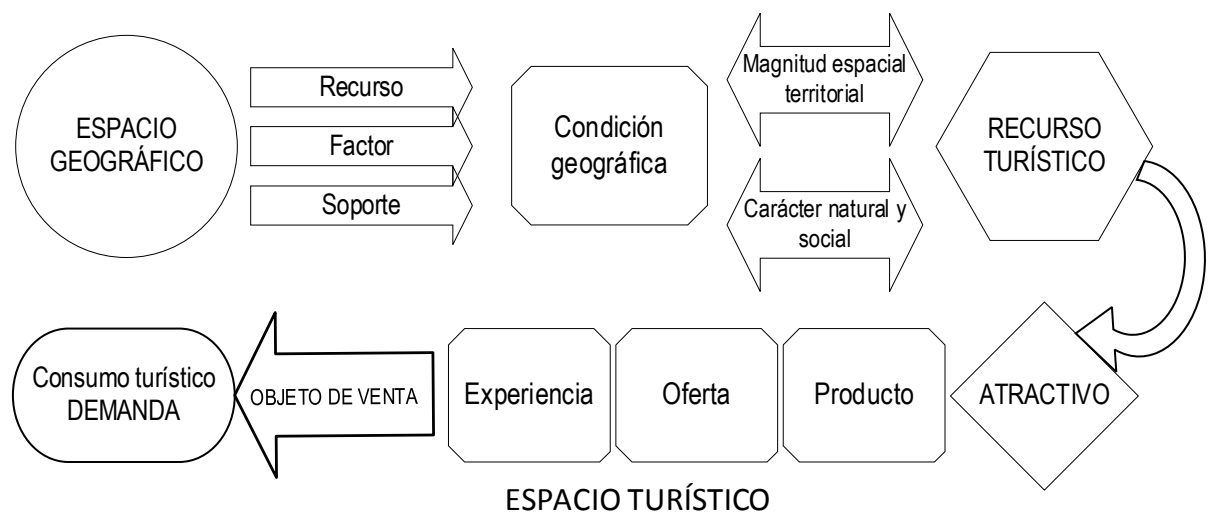

Fuente: Elaboración propia.

El diagrama 1 modela esquemáticamente los preceptos antes expuestos e incorpora, la mirada espacial considerando el sistema de relaciones naturaleza sociedad y el territorio por la connotación política administrativa. También agrega la experiencia turística, que implica la vivencia personal diferenciada en el consumo, a partir del producto y la oferta; dependiendo de cómo se configure será el nivel de demanda.

Pearce (2014) aportó seis áreas de la geografía del turismo: patrones de distribución de la oferta, la demanda, los centros vacacionales, los movimientos 
y flujos, el impacto y los modelos de desarrollo del espacio turístico (Vera et al, 1997:51). El turismo es espacio movimiento, el desplazamiento consume tiempo, el viaje es transición de espacios y depende de qué recursos se valoran y cómo se valoran (Vera et al, 1997:51; Barrado, 2011:48, Pinassi y Ercolani, 2017:43).

El estudio territorial del turismo considera variables temáticas cuantificables (García, 2015:36). Estas son: infraestructura, entornos y sus transformaciones, relación población residente y turistas, planificación territorial y urbanística, con un comportamiento espacial. Para Buzai, Baxendale y Cruz, (2009:2) significa "conocer para hacer, para actuar, para construir, para modificar".

Las categorías espacio, territorio y tiempo, están presentes en el continuo desarrollo de las TIC y de las ciencias geográficas a través de los SIG. Estos últimos, armonizan hardware $y$ software, almacenando datos para crear mapas basados en computadora que, muestran la ubicación y los atributos de los objetos de interés para la toma de decisiones (Hess et al, 2004:198) Los SIG junto a los Global Positioning System (GPS) precisan la medición del tiempo y la ubicación de la actividad, facilitan herramientas tecnológicas, metodológicas y conceptuales para el turismo (Shoval, et al, 2014:3; Zhou, et al, 2019:2).

Las implicaciones para los turistas, radica en recibir más información fuera y dentro del destino, el conocimiento espacial provee una experiencia turística significativa (Tussyadiah y Zach, 2012:3). Las tecnologías citadas ayudan a tomar decisiones a los visitantes, también a gestores y comunidades que se auxilian del contenido para configurar los productos, ofertas y todo el itinerario del consumo del turista.

Lo anterior se ejemplifica en el SIG turístico de Islas Canarias (García, 2015:36) que enfatiza aspectos objetivos. Habría que incluir variables subjetivas, relacionadas con el turista y su satisfacción. El cuadro 1, expresa las variables seleccionadas (capas temáticas) para el análisis general $\mathrm{y}$ específico turístico.

\section{Cuadro 1 \\ Ejemplos de variables seleccionadas en capas temáticas de un SIG.}

\begin{tabular}{ll}
\hline Modelos & Variables seleccionadas (capas temáticas) \\
\hline Vectorial & $\begin{array}{l}\text { De análisis general: Distribución de la población y caracterización geodemográfica por unida- } \\
\text { des territoriales, uso residencial, ordenación territorial y urbanístico. }\end{array}$ \\
& $\begin{array}{l}\text { De análisis turístico: Delimitación territorial turística, localización y caracterización de la } \\
\text { infraestructura turística, atracciones registradas (atributos: fecha de creación; calificación de } \\
\\
\text { la atracción; fotos añadidas, número y nacionalidades de los visitantes a la atracción, com- } \\
\text { portamiento de la demanda según territorios turísticos, percepciones). }\end{array}$ \\
\hline Ráster & De análisis general: ocupación del suelo, cambios en usos y coberturas del suelo, ordena- \\
& ción. \\
& De análisis turístico: Procesos de crecimiento y transformación del entorno urbano-turístico. \\
\hline
\end{tabular}

Fuente: Elaboración propia a partir de García, (2015:36), Rosa-Jiménez C. et al, (2016:86) 
Combinada con la tecnología SIG, la computación en la nube e inteligencia artificial, distribuye información turística relevante en Internet. Realzan Rosa-Jiménez et al, (2016:88) las herramientas Web (OpenStreetMap, sitio web Geofabrik, TripAdvisor) para analizar, aspectos objetivos de los recursos, espacios y destinos turísticos; y subjetivos de turistas. Por esto, según Kim, et al, (2017:362) se ha generado un cambio en los patrones de comportamiento de los turistas.

Shoval (2018:911) destaca las tecnologías de rastreo en sensores integrados (GPS, posicionamiento móvil, mensajes de redes sociales geocodificadas, Bluetooth, acelerómetro, giroscopio, brújula, sensor de luz, micrófono y cámara). Señalan Perilla y Mas (2020:2) "cuan poderoso es el Google Earth Engine, que vincula el potencial de los datos masivos y la eficacia del procesamiento en la nube almacenando imágenes de varios sensores (Landsat, Sentinel, MODIS, entre otros)". Todas facilitan la cartografía en tiempo real de información cuantitativa o cualitativa.

El análisis bibliométrico de la base de datos Scopus mediante el software VOSviewer y las palabras clave: sistemas de información geográfica y turismo; revelan las relaciones entre ambos conceptos. Desde 2011 a enero de 2020 un total de 302 documentos abordaron los nexos referidos como eje principal. Hasta ese momento, la mayor cantidad de artículos se publicaron en 2018 y ya dos correspondían a 2020. Las Ciencias Ambientales, de la Computación e Ingenierías, fueron las que más trataron el tema. Resaltan por cantidad de artículos (en paréntesis): Aminu, M. (5), Matori, A.N. (5), Yusof, K.W. Show (4), Boori, M.S. (3), Bozorgnia, D.S(3).
Hasta 2017 destacan los temas: planificación del turismo sostenible, modificación de la cobertura del suelo debido al turismo, evaluación del potencial ecoturístico. Prima el enfoque ambiental y para la gestión, no en función de la experiencia del turista. Durante 2018 y 2020 se aborda: turismo inteligente, inteligencia artificial y sistema SIG, rastreo con Google, relacionados con el ser humano y la salud, las actividades recreativas, las redes sociales, criterios múltiples para el diseño de rutas de viaje, evaluación de riesgos, aplicaciones SIG en turismo, análisis multicriterio y SIG, herramienta Web GIS (SIG en inglés). Las cuatro agrupaciones definidas, giraron alrededor de: SIG, turismo, turismo sostenible y uso del suelo.

En síntesis, se destaca el rol de los SIG y su armonización con otras herramientas TIC al abordar la espacialidad y territorialidad del turismo, orientado a nuevos paradigmas en la toma decisiones de gestores y comunidades turísticas para la satisfacción del cliente, sus motivaciones y experiencias.

\section{Gestión integral de destinos turísticos y calidad de las relaciones múltiples}

La interpretación científica del marco conceptual de destinos turísticos, permitirá aplicar los principios en la práctica y la gestión para mejores resultados. Señala Pearce (2014:141) que la naturaleza y el alcance de los conceptos utilizados pueden determinar el enfoque de la gestión, quién o qué se gestiona, dónde están las prioridades de gestión y dónde las soluciones. También, propone un marco conceptual integrador de destinos, que incluye 
cinco conceptos: distritos industriales, clúster, redes, sistemas y construcciones sociales; atravesados por tres dimensiones principales: geográfica, modo de producción y dinámica.

Un distrito industrial es una entidad socio-territorial con una comunidad de personas y un agrupamiento de empresas en un área natural e históricamente limitada Becattini (1990:38), estos aspectos son válidos para el destino turístico. El análisis clúster de Porter (1998:79) es una forma organizativa espacial entre los mercados independientes y las jerarquías, o la integración vertical; pero de acuerdo con Fabry (2009:60) en turismo la producción y el consumo ocurren simultáneamente.

Las redes, se componen de nodos y conexiones; en la sociedad los nodos son los actores y las conexiones los vínculos sociales (Pearce, 2014:141). En el turismo las relaciones entre los involucrados persiguen brindar experiencias. Destaca Ballina (2020:4) que el enfoque de destino en red, es el modelo integrado más desarrollado actualmente, en cuyo seno ocurre la cocreación de experiencias.

Un sistema como grupo de elementos interdependientes que juntos forman una sola estructura funcional (Weaver y Oppermann 2000:23 citado por Pearce, 2014:141). El sistema turístico está conformado por cuatro componentes principales; la demanda, la oferta, los operadores y el espacio turístico (Sancho, 1998:47); donde se generan flujos de turistas que salen y regresan (Leiper,1979:397). La relación de esos subsistemas en el espacio y territorio, con una imagen identidad propia incluyendo las comunidades anfitrionas, es el destino turístico. Coincidiendo con Ballina (2020:2) este es contenedor principal del producto final que consume el turista, como un todo o en partes, dentro de un espacio territorial y una imagen afín.

Las construcciones sociales enfatizan la transformación, la participación y los significados que las personas, dan a sus vidas y a los lugares donde habitan; en tanto, experiencias para los turistas. Las relaciones identificadas entre los mismos miembros de la comunidad generan alternativas y el cambio; mientras las relaciones entre clientes internos e internos son clave en la medición de la calidad (Baquero y Cárdenas, 2020:243; Angamarca, Díaz y Martínez, 2020:385). También, la interactividad y la personalización tienen importancia crucial para la experiencia turística (Jeong \& Shin, 2020:3; Filho, Silva \& da Silva 2019:108) la participación, la confianza y el compromiso, se incorporan en el modelo como calidad de la relación.

La certificación de la calidad, requiere de todas las relaciones ya citadas; coincidiendo con SánchezValdés, Nava-Rogel, y Delgado-Cruz (2021:365); Ormaza y Guerrero-Baena (2021)es un elemento competitivo y factor estratégico, respecto a la percepción del turista y el posicionamiento, resultado de una gestión integral.

Un antecedente de gestionar integralmente el destino turístico procede del marketing, a través de la "Organización de Marketing de Destino" (DMO siglas en inglés). Choi \& Cai (2017:10) la conceptualiza como un enfoque holístico y relacional para el marketing de destino integrado. Wang y Fesenmaier (2006) y Wang y Xiang (2007) citados por Elbe, Hallén, \& Axelsson, (2009:285), modelan, describen y explican esas alianzas que, limitadas al marketing, fomentan la cooperación y la integración; expresada 
en la relación de los componentes del producto turístico.

El modelo integral de Dwyer, et al, (2014:3-5) comprende como principales determinantes: recursos dotados naturales o culturales, recursos creados y de apoyo; gestión de destinos a partir de alianzas entre el sector público y privado; condiciones de demanda (conciencia, percepción y preferencias); condiciones del entorno y tendencias, influyentes en flujos turísticos y la competitividad del destino.

Recientemente, la OMT (2019a:12) aboga por las Organizaciones de Gestión de Destinos (OGD) integrales, dejando atrás la mirada estrecha de la DMO. Definiéndola como "la principal entidad organizativa formada por diversas autoridades, agentes y profesionales, y facilita las alianzas dentro del sector turístico con miras a un proyecto colectivo para el destino".

Otros autores agregan valor a la OGD, en cuanto, proporcionan un marco integral y estable, agrupando políticas sectoriales, directrices y líneas de actuación con interconexiones (PulidoFernández y López-Sánchez, 2016:172). Además, junto a las comunidades, facilitan efectivas formas de colaboración (Paddison, \& Biggins, 2017:2). Asignando a los ciudadanos un papel activo en el desarrollo de políticas y en la distribución de sus beneficios; equilibran las relaciones entre el desarrollo turístico, los procesos de planificación y todas las partes interesadas (Hsu \& Gu, 2010:60; Garrod et al, 2012; Le Feuvre et al, 2015; Paddison \& Biggins, 2017:5). Dichos paradigmas buscan el modelo turístico sostenible con énfasis en la calidad de las relaciones colectivas para los procesos de gestión en el destino turístico.

La evolución de la planificación turística en España se ha basado en la cooperación entre las administraciones central, autonómica y local (Ivars-Baidal y Vera Rebollo, 2019:10). Allí, un desafío es, el enfoque holístico para la gestión de destinos, en el que los criterios sostenibles son una prioridad que se desglosan en la gestión integrada de destinos turísticos y la corresponsabilidad entre los sectores público y privado del turismo (Valls \& Sardá, 2009:47).

A continuación, una forma de proporcionar la integración, es a través de la aplicación la TIC. En los destinos inteligentes (López de Ávila et al, 2015:149; Dolan \& Kemper, 2019:42) la tecnología se encuentra integrada en todos los ámbitos con las personas y sus acciones. Ello implica, participación social en la toma de decisiones, transparencia, énfasis en la colaboración, liderazgo, comunicación asertiva, intercambio de datos y responsabilidad.

\section{Gestión inteligente de destinos turísticos, más que tecnología}

Muchas de las actividades que realiza el ser humano en la actualidad, son auxiliadas por las TIC; porque viabilizan las diversas tareas de la sociedad. Así, inteligente es una categoría de productos, servicios y sistemas, donde las TIC vanguardias ocupan un papel clave (Höjer \& Wangel, 2015:336) ya que reducen el costo, el tamaño de la capacidad informática, facilitando nuevas ofertas y modelos de negocios (Williams, Rodríguez, \& Makkonen, 2020; Shen, Sotiriadis \& Zhang, 2020; Montecinos, 2021).

La inteligencia se asocia según Harrison et al, (2010) citado por Gretzel, Sigala, Xiang \& Koo (2015:42), con la 
explotación de datos operativos del mundo, a veces en tiempo real, que son integrados, compartidos y usados en análisis complejos; modelado, optimización y visualización para tomar mejores decisiones operativas. Los rasgos comunes a los territorios inteligentes son: los diseña la comunidad, tecnología digital, conexiones con redes, innovación, coherencia de gobernanza, cohesión y desarrollo social y responsabilidad ambiental (López de Ávila, et al, 2015:112).

Señala la OMT (2019a:11), que los destinos turísticos inteligentes se sustentan en cinco pilares: gobernanza, innovación, tecnología, accesibilidad, sostenibilidad. Agrega
Salessi, (2017:222) que se nutren de datos, gobierno abierto, y participación ciudadana, con una dinámica que se reconstruye y retroalimenta constantemente, compartiendo las buenas prácticas. El análisis del concepto destino turístico inteligente a partir de: López de Ávila et al, (2015:104) Gretzel et al, (2015:3), Fernandes (2017:62) citado por Santos-Júnior et al, (2019:30) SEGITTUR. (2018:4), UNE 178501 citado por Valderrama (2018), SantosJúnior et al, (2019:30); permite identificar grupos de palabras más repetidas y modelarlas en un esquema, la diagrama 2 sintetiza la interpretación realizada por la autora de este artículo.

\section{Diagrama 2 Interrogantes sobre destinos turísticos inteligentes}

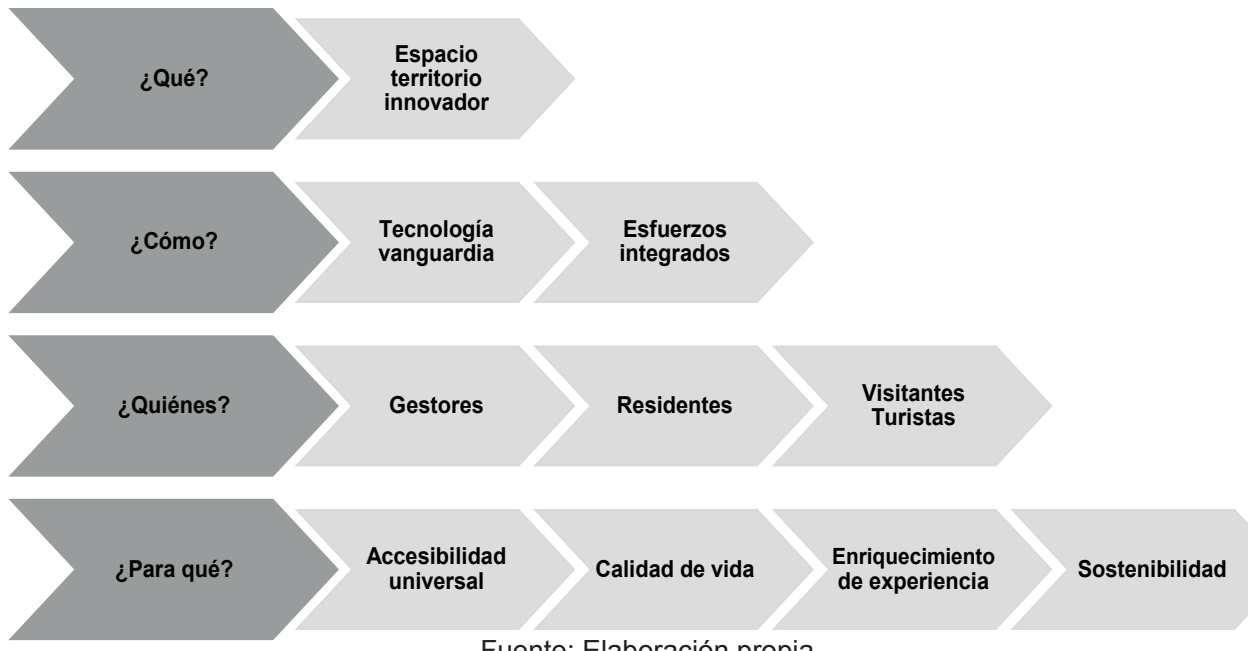

Fuente: Elaboración propia 
Se asume un destino turístico inteligente como un espacio territorio innovador que utiliza tecnología de avanzada en condiciones de esfuerzos integrados de gestores y residentes para la accesibilidad universal, la calidad de vida y el enriquecimiento de la experiencia del visitante; todo enfocado en la sostenibilidad total.

Los destinos turísticos inteligentes van más allá de la tecnología, aunque implique, profusión de datos de alto volumen, velocidad y variedad (big data), diversidad de sistemas tecnológicos (Hunter, Chung, Gretzel \& Chulmo, 2015:106; Filho, Silva \& da Silva 2019:108). La innovación (crear algo nuevo como un todo) agrega una perspectiva más integral (Williams et al, 2020:9). Además, la mejora de experiencias turísticas y la sostenibilidad son resultados principales de esta entidad; abarcando esferas del funcionamiento natural social, como: eficiente consumo energético y de agua, tratamiento de residuos, facilidades para personas con discapacidad, equilibrio entre satisfacción de turistas y calidad de vida de los residentes (Wang, Li X., \& Li Y. 2013:61; Ivars-Baidal, CeldránBernabeu, Mazón \& Perles-Ivars, 2017:11; Celdrán Bernabéu, 2018:4; Filho et al, 2019:99).

El empleo de la TIC en el turismo ha transitado desde el Turismo tradicional (80s) con sistemas globales de distribución y centrales de reserva; pasando por e-turismo y la web 2.0 hasta la actualidad Turismo inteligente (Cuadro 2).

\section{Cuadro 2}

\section{Turismo inteligente vs. e-turismo}

\begin{tabular}{lll}
\hline Criterios de comparación & e-Turismo (e-Tourism) & $\begin{array}{l}\text { Turismo inteligente (Smart } \\
\text { Tourism) }\end{array}$ \\
\hline Esfera & Digital & Puente digital y físico \\
\hline Tecnología del núcleo & Sitios web & $\begin{array}{l}\text { Sensores y teléfonos inteli- } \\
\text { gentes }\end{array}$ \\
\hline Fase de viaje & Pre y post viaje & Durante el viaje \\
\hline Alma & Información & Big data \\
\hline Paradigma & Interactividad & $\begin{array}{l}\text { Co-creación mediada por tec- } \\
\text { nología }\end{array}$ \\
\hline Estructura & Cadena de valor / intermediarios & $\begin{array}{l}\text { Ecosistema social } \\
\text { Intercambiar }\end{array}$ \\
$\begin{array}{ll}\text { B2B (Busissnes to Busissnes o B-to-B) } \\
\text { Negocio a negocio. Comercio electrónico } \\
\text { Internet }\end{array}$ & $\begin{array}{l}\text { Colaboración público-priva- } \\
\text { da-consumidor (Business to } \\
\text { (Business-to-Consumer o B to C) negocio } \\
\text { a consumidor. C2C (Consumer to Con- } \\
\text { procesos de negociación entre } \\
\text { empresas y el gobierno a través } \\
\text { del uso de Internet. (Business to } \\
\text { Government to Consumer o B } \\
\text { to G to C) }\end{array}$ \\
\hline
\end{tabular}


Las críticas al enfoque inteligente, son: grandes empresas tecnológicas beneficiadas, favorecimiento a la privatización, dependencia tecnológica, incógnitas sobre la privacidad, despolitización falsa de la gestión (IvarsBaidal y Vera, 2019:10).

España ha sido abanderada; la Secretaría de Estado del Turismo a través de SEGITTUR han desarrollado una metodología que, según Martínez (2019:38) utiliza los enfoques multidisciplinares e integradores, unido a las dimensiones de un buen gobierno, sostenibilidad, accesibilidad y de forma transversal, la tecnología. Un avance distintivo es que la Asociación Española de Normalización y Certificación (AENOR), ha normado la gestión de los destinos turísticos inteligentes. Señala Höjer \& Wangel, (2015:336) que la opción normativa, usa el concepto instrumentalmente.

Esta revisión teórica contrastada y la experiencia vivida en Palma de las Islas Baleares, durante septiembre octubre de 2019, permiten modelar la gestión integral e inteligente del destino turístico desde la perspectiva espacio territorio (diagrama 3) ayudando a comprender pautas principales que son aplicables a otros contextos.

\section{Diagrama 3 \\ Espacio territorio en la gestión integral e inteligente del destino turístico}

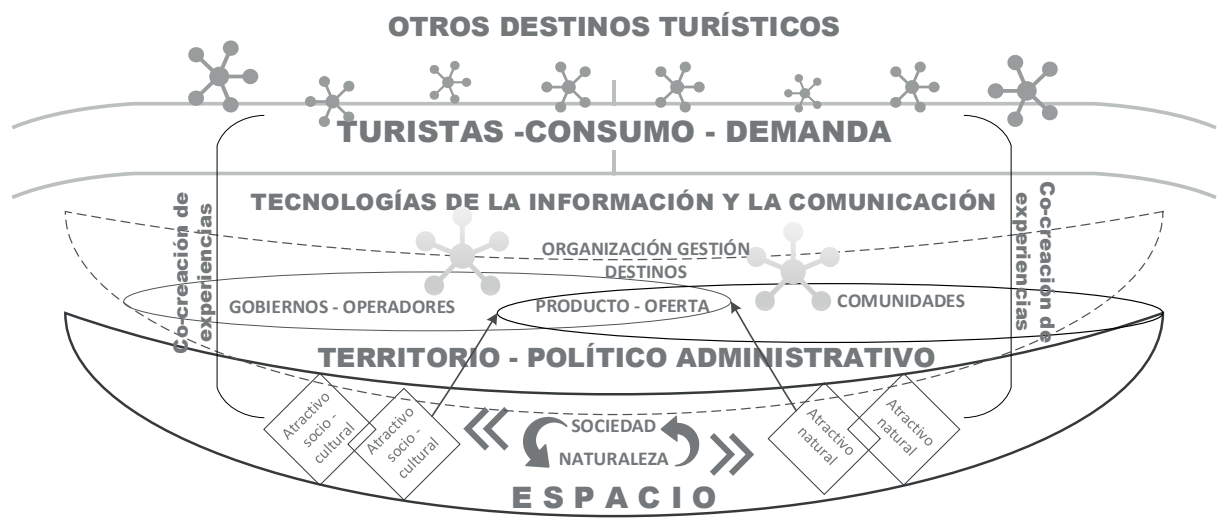

Fuente: Elaboración propia

Palma es la ciudad capital de las Islas Baleares, se asienta en un espacio litoral insular, colmado de atractivos naturales y culturales. Allí, los residentes y visitantes viven al ritmo de la inteligencia del destino. La sociedad se configura en función de los tiempos programados para distintos medios de transportes 
Perspectiva espacio - territorio en la gestión integral e inteligente de destinos turísticos

y de la red que tejen los recorridos de líneas y vías, que convergen en el centro histórico-comercial.

La orientación, comunicación, desplazamientos, planificación de las compras y el consumo de la gran variedad de productos y servicios; fluyen a través de la tecnología. No importa el segmento de edad, desde los más jóvenes hasta las personas de la tercera edad, organizan la vida rutinaria en el institucionalizado sistema que la gobernanza controla y que armoniza con el uso de las TIC.

La fusión de servicios de todo tipo se proyecta a la accesibilidad universal. En mapas colmados de información, frecuentemente en tiempo real, se registran datos sobre la calidad, cantidad, tamaños, formas, colores, sabores (de acuerdo con los productos y ofertas) en la nube y aplicaciones móviles. Todo, es resultado de las relaciones armónicas entre las partes interesadas, lo que hace enriquecedora la experiencia de turistas y residentes, aunque estos últimos sienten el peso del exceso de visitantes.

\section{Conclusiones}

La perspectiva espacio territorio está presente en la gestión de destinos turísticos porque ellos soportan, aportan y son parte del sistema turístico. El espacio turístico contiene recursos atractivos (naturales y socioculturales), productos, ofertas y experiencias; el límite territorial representa gobernanza y todo lo que conlleva.

En las condiciones actuales cuando las TIC minan todas las esferas, la gestión turística requiere de ellas, para expresar el carácter espacial territorial de la actividad; en particular a través de los SIG y de la diversidad de herramientas de rastreo que, con precisión informan de ubicación, accesibilidad, atributos y desplazamientos en el tiempo; sobre las personas, objetos y procesos involucrados.

La calidad de las relaciones múltiples presentes en organizaciones, redes, sistemas, constructos sociales expresan interdependencias dinámicas en el tiempo y espacio; aspecto clave en la gestión integrada e inteligente de destinos turísticos. Dicha gestión utiliza la tecnología de avanzada y la innovación, para incidir en la calidad de vida de los residentes, crear experiencias inolvidables en los turistas y aportar a la sostenibilidad en los espacios y territorios donde se desarrolla el turismo.

La experiencia de España no es un patrón rígido, muestra un ejemplo de funcionamiento. Se requiere de investigación continua que llene los vacíos de la gestión integrada e inteligente de los destinos turísticos considerando la espacialidad y territorialidad, porque a más de todo lo argumentado, esta perspectiva es base de la singularidad de cada lugar, a partir de la cual se desarrollan las relaciones múltiples.

\section{Referencias bibliográficas}

Angamarca, G. R., Díaz, Y., y Martínez, C. (2020). Calidad percibida por los clientes de los hoteles de la provincia Los Ríos, Ecuador. Revista de Ciencias Sociales (Ve), XXVI(4), 380-393. https://doi.org/10.31876/ rcs.v26i4.34669

Ballina Ballina, F. J. (2020). El protocolo "network" aplicado al estudio de los destinos turísticos: el caso de Gijón (España). Estudios Geográficos, 81(288), e041. https://doi. org/10.3989/estgeogr.202056.056

Baquero, D., y Cárdenas, S. (2020). 
Redes y actores: Un avance en las relaciones de poder en la comunicación organizacional. Revista de Ciencias Sociales (Ve), $X X V I(4), \quad 232-245 . \quad$ https://doi. org/10.31876/rcs.v26i4.34660

Barrado, D. A. (2011). Recursos territoriales y procesos geográficos: el ejemplo de los recursos turísticos. Estudios Geográficos, LXXII(270), 35-58. $\quad$ https://doi.org/10.3989/ estgeogr.201102

Becattini, G. (1990). The Marshallian Industrial District as a Socioeconomic Notion. In Industrial Districts and Inter-Firm Co-operation in Italy, edited by F. Pyke. Geneva: International Institute for Labour Studies, pp. 37-51.

Bigné, J. E., Font X., Andreu L. (2000). Marketing de destinos turísticos: análisis y estrategias de desarrollo. Editorial ESID. Madrid, Es.

Butler, R.W. (1980). The concept of a tourist area cycle of evolution: Implications for management of resources. University of Western Ontario Canadian Geographer, XXIV, 1.

Buzai, G.D. (2009). Consideraciones sobre el rol científico de los SIG a dos décadas de pensamientos sobre la Geografía de Peter Gould, Huellas. Universidad Nacional de La Pampa, $n^{\circ} 12$, pp. 265-281.

Celdrán, M. A. (2018). Nuevos escenarios para la planificación y gestión del turismo. El enfoque destinos turísticos inteligentes (Summary of Doctoral dissertation, Universidad de Alicante, Spain). Boletín de la Asociación de Geógrafos Españoles, 79, 2727, 1-12.

Choi, S., \& Cai, L. A. (2017). The role of relationship quality in integrated destination marketing. Journal of
Travel \& Tourism Marketing, 35(5), 541-552. https://doi.org/10.1080/105 48408.2017.1403999

Dolan, R., Seo, Y., \& Kemper, J. (2019). Complaining practices on social media in tourism: A value co-creation and co-destruction perspective. Tourism Management, 73, 35-45. https://doi.org/10.1016/j. tourman.2019.01.017

Dwyer, L., Cvelbar, L. K., Mihalič, T., \& Koman, M. (2014). Integrated destination competitiveness model: Testing its validity and data accessibility. Tourism Analysis, 19(1), 1-17. https://doi.org/10.3727/108354 214X13927625340073

Elbe, J., Hallén, L., \& Axelsson, B. (2009). The destination-management organisation and the integrative destination-marketing process. International Journal of Tourism Research, 11(3), 283-296. https://doi. org/10.1002/jtr.695

Fabry, N. (2009). Clusters de tourisme et compétivité des acteurs: une solution pour conforter le tourisme d'affaires et événementiel parisien? In Destinations et territoires: Coprésence à l'œuvre, edited by J.-P. Lemasson and $\mathrm{P}$. Violier. Québec: Editions Téoros/ Presses de l'Université de Québec, pp. 55-68.

Filho L. M., Silva J. C., da Silva D. S. (2019). Percepções e perspectivas de destino turístico inteligente: um estudo de caso com a Secretaria Municipal de Turismo em Natal. Turismo: Estudos \& Práticas, Mossoró/RN, 8(1). http://periodicos. uern.br/index.php/turismo/article/ view/3855

Font, M. (2002). Diferenciación geográfico ambiental del municipio y la ciudad de Santa Clara para la vigilancia en salud. Tesis presentada en opción al título de Doctor en 
Perspectiva espacio - territorio en la gestión integral e inteligente de destinos turísticos

Ciencias Geográficas. Universidad de La Habana.

García, J. (2015). El análisis del impacto territorial del tercer boom turístico en Canarias (España) a través de la aplicación de un SIG. Cuadernos de Turismo, (36), 219-245. https://dx.doi. org/10.6018/turismo.36.230971

Garrod, B., Fyall, A., Leask, A., \& Reid, E. (2012). Engaging residents as stakeholders of the visitor attraction. Tourism Management, 33(5), 11591173. $\quad$ https://doi.org/10.1016/j. tourman.2011.11.014

Gretzel, U., Sigala, M., Xiang, Z., \& Koo, C. (2015). Smart tourism: foundations and developments. Electronic Markets, 25(3), 179-188. https://doi. org/10.1007/s12525-015-0196-8

Hess, R. L., Rubin, R. S., \& West, L. A. (2004). Geographic information systems as a marketing information system technology. Decision Support Systems, 38(2), 197-212. https://doi. org/10.1016/s0167-9236(03)00102-7

Höjer, M., \& Wangel, J. (2015). Smart Sustainable Cities: Definition and Challenges. In L. M. Hilty \& B. Aebischer (Eds.), ICT Innovations for Sustainability, Advances in Intelligent Systems and Computing (pp. 333-349). New York: Springer International Publishing Switzerland. https://doi.org/10.1007/978-3-319$\underline{09228-7 \quad 20}$

Hsu, C. H. C., \& Gu, Z. (2010). Ride on the gaming boom: How can Hong Kong, Macau and Zhuhai join hands to develop tourism in the region? Asia Pacific Journal of Tourism Research, 15(1), 57-77. https://doi. org/10.1080/10941660903510057

Hunter, W. C., Chung N., Gretzel, U., y Chulmo Koo. (2015). Constructivist Research in Smart Tourism. Asia Pacific Journal of Information
Systems, 25(1). http://dx.doi. org/10.14329/apjis.2015.25.1.105

Ivars-Baidal, J. A., \& Vera Rebollo, J. F. (2019). Planificación turística en España. De los paradigmas tradicionales a los nuevos enfoques: planificación turística inteligente. Boletín de la Asociación de Geógrafos Españoles, 82, 2765, 1-31. http:// dx.doi.org/10.21138/bage. 2765

Ivars-Baidal, J. A., Celdrán-Bernabeu, M. A., Mazón, J.-N., \& Perles-Ivars, Á. F. (2017). Smart destinations and the evolution of ICTs: a new scenario for destination management? Current Issues in Tourism, 1-20 https://doi.or g/10.1080/13683500.2017.1388771

Jeong, M., \& Shin, H. H. (2020). Tourists' experiences with smart tourism technology at smart destinations and their behavior intentions. Journal of Travel Research, 59(8), 1464-1477. https://doi. org/10.1177/0047287519883034

Kim, K., Park, O. -., Yun, S., \& Yun, H. (2017). What makes tourists feel negatively about tourism destinations? application of hybrid text mining methodology to smart destination management. Technological Forecasting and Social Change, 123, 362-369. https://doi. org/10.1016/j.techfore.2017.01.001

Le Feuvre, M., Medway, D., Warnaby, G., Ward, K., \& Goatman, A. (2015). Understanding stakeholder interactions in urban partnerships. Cities, 52, 55-65. https://doi. org/10.1016/i.cities.2015.10.017

Leiper, N. (1979). The Framework of Tourism: Towards a Definition of Tourism, Tourist, and the Tourist Industry. Annals of Tourism Research, 6(4), 390-407.

López de Ávila A., Lancis E., García S., Alcantud A., García B., Muñoz N. 
(2015). Informe destinos turísticos inteligentes: construyendo el futuro. Pautas de actuación para la conversión en destino turístico inteligente: Guía práctica. 207pp. (SEGITTUR, S.A.). Madrid, septiembre de 2015.

Martínez, E. (2019). Destinos Turísticos Inteligentes. El futuro del turismo va a depender de nuestra capacidad de adaptarnos y anticiparnos a los cambios. Agenda de la Empresa, XXIII(248). 37- 40. https://bit. ly/3dTEkl5

Montecinos, E. (2021). Cuarta revolución industrial y la administración pública en América Latina. Revista Venezolana de Gerencia (Ve), XXVI(93), 10-32.

OMT. (2019a). Directrices de la OMT para el fortalecimiento de las organizaciones de gestión de destinos (OGD) - Preparando las OGD de cara a nuevos retos, OMT, Madrid. https:// doi.org/10.18111/9789284420933

Organización Mundial del Turismo(OMT). (2019). Definiciones de turismo de la OMT. OMT, Madrid. https://doi. org/10.18111/9789284420858

Ormaza, M. G. y Guerrero-Baena, M. D. (2021). Gestión de calidad y crecimiento empresarial: Análisis bibliométrico. Revista Venezolana de Gerencia (Ve), XXVI(93), 318-333.

Paddison, B., \& Biggins, R. (2017). Advocating community integrated destination marketing planning in heritage destinations. Journal of Marketing Management, 33(9-10), 835-857. https://doi.org/10.1080/026 $\underline{\text { 7257X.2017.1329226 }}$

Pearce, D. G. (2014). Toward an Integrative Conceptual Framework of Destinations. Journal of Travel Research, 53(2) 141 -153. https:// doi.org/10.1177/0047287513491334
Perilla, G. A. y Mas, J.F. (2020). Google Earth Engine (GEE): una poderosa herramienta que vincula el potencial de los datos masivos y la eficacia del procesamiento en la nube. Revista Investigaciones Geográficas, 101. https://doi.org/10.14350/rig.59929

Pinassi, C. A. y Ercolani P. S. (2017). Turismo y espacio turístico: un análisis teórico conceptual desde la Ciencia Geográfica. Rev. Anais Bras. de Est. Tur./ ABET, 7(1), 42-61.

Porter, M. A. (1998). Clusters and the New Economics of Competition. Harvard Business Review, 77-90.

Pulido-Fernández, J. I., \& LópezSánchez, Y. (2016). La cadena de valor del destino como herramienta innovadora para el análisis de la sostenibilidad de las políticas turísticas. Innovar, 26(59), 155-176. https://doi.org/10.15446/innovar. v26n59.54369

Rosa-Jiménez, C., Reyes-Corredera, S., \& Nogueira-Bernárdez, B. (2015). New possibilities of GIS for mapping a mature destination: a case in Benalmádena, Spain. Anatolia, 27(1), 82-90. https://doi.org/10.1080 /13032917.2015.1083211

Salessi M. L. (2017). Destinos Turísticos Inteligentes: Una perspectiva desde la inclusión social y la participación comunitaria. Actas del Seminario Internacional Destinos Turísticos Inteligentes. Universidad de Alicante, 26 y 27 de octubre de 2017. Pp. 215. Consultado 18 de octubre de $2019 . \quad$ https://rua.ua.es/dspace/ bitstream/10045/70141/5/ActasSeminario-Destinos-TuristicosInteligentes.pdf

Sánchez-Crispín, Á., Alvarado-Sizzo, I., \& Propin-Frejomil, E. (2018). Territorial structure of tourism in Santiago, Nuevo León, México. Investigaciones Geográficas, 
Perspectiva espacio - territorio en la gestión integral e inteligente de destinos turísticos

\section{https://doi.org/10.14350/rig.59620}

Sánchez-Valdés, A., Nava-Rogel, R. M. y Delgado-Cruz, A. (2021). Motivaciones de empresas turísticas para certificarse en calidad. Evidencia empírica en el Estado de México. Revista Venezolana de Gerencia (Ve), XXVI(93), 318-333.

Sancho, A. y otros. (1998). Introducción al Turismo. OMT. http://0-wwwe--unwto-org.Ilull.uib.es/doi/ pdf/10.18111/9789284402694

Santos-Júnior, A.; Augusto-Biz, A.; Almeida-García, F.; Mendes-Filho, I. (2019). Entendiendo la gobernanza de los destinos turísticos inteligentes: el caso de Florianópolis - Brasil. International Journal of Information Systems and Tourism (IJIST), 4(1), 29-39.

Secretaría de Estado de TurismoEspaña (SETUR). (2019). ¿Qué es el SICTED? Sistema Integral de Calidad Turística en Destinos. Ministerio de Industria, Energía y Turismo. Madrid. https://www.calidadendestino.es/ documentos/2019 1003 Que es el SICTED.pdf

SETUR - SEGITTUR. (2018). La estrategia española de destinos turísticos inteligentes. Jornadas Europeas, sostenibilidad y territorios inteligentes. https://docplayer. es/95511513-La-estrategiaespanola-de-destinos-turisticosinteligentes.html

Shen, S., Sotiriadis, M., \& Zhang, Y. (2020). The influence of smart technologies on customer journey in tourist attractions within the smart tourism management framework. Sustainability (Switzerland), 12(10) https://doi.org/10.3390/su12104157

Shoval, N. (2018). Sensing tourists: Geoinformatics and the future of tourism geography research. Tourism
Geographies, 20(5), 910-912. https:// doi.org/10.1080/14616688.2018.143 $\underline{7768}$

Shoval, N., Kwan, M-P., Reinau, K. H., \& Harder, H. (2014). The shoemaker's son always goes barefoot: Implementations of GPS and other tracking technologies for geographic research. Geoforum, 51(1), 1-5.

Simancas, M., Peñarrubia, M. P., \& Temes, R. (2018). The land classification: A key issue in the prohibition of holiday rental in the coastal tourist areas of the Canary Islands. https://doi.org/10.30827/ cuadgeo.v57i1.5666

Suarez, N. y otros. (2002). Informe Técnico Campos de Acción Académicos. Espacio y Territorio. Bitacora, 6(1), 33- 76.

Tussyadiah, I.P., y Zach, F. (2012). The Role of Geo-Based Technology in Place Experiences. Annals of Tourism Research, 39(2), 780-800.

Valderrama, J. Á. (2018). ¿Cómo ser un destino turístico inteligente? AENOR, $n^{\circ} 339$, julio-agosto. 2018. Dirección de Operaciones de Evaluación de la Conformidad. https://revista.aenor. com/339/como-ser-un-destinoturistico-inteligente.html

Valls, J. F., \& Sardá, R. (2009). Tourism expert perceptions for evaluating climate change impacts on the Europa Mediterranean tourism industry. Tourism Review, 64(2), 41-51. https://doi. org/10.1108/16605370910963518

Vera Rebollo J. F., López Palomeque F., Marchena Gómez M., Antón S. (1997). Análisis territorial de turismo: una nueva geografía del turismo. Ariel.

Wang, D., Li, X. Robert, \& Li, Y. (2013). China's "smart tourism 
pp. 915-931

Revista Venezolana de Gerencia, Año 26 No. 94, 2021

destination" initiative: A taste of the service-dominant logic. Journal of Destination Marketing \& Management, 2(2), 59-61. https:// doi.org/10.1016/j.jdmm.2013.05.004

Williams, A. M., Rodríguez, I., \& Makkonen, T. (2020). Innovation and smart destinations: Critical insights. Annals of Tourism Research,
83 annals.2020.102930

Zhou, Z., Feng, Z., \& Li. (2019). Individualized Tour Route Plan Algorithm Based on Tourist Sight Spatial Interest Field. ISPRS International Journal of GeoInformation, 8(4), 192. https://doi. org/10.3390/ijgi8040192. 